\title{
Subharmonics and Chaos in Switched Reluctance Motor Drives
}

\author{
J. H. Chen, K. T. Chau, Member, IEEE, C. C. Chan, Fellow, IEEE, and Quan Jiang
}

\begin{abstract}
In this paper, the investigation of the nonlinear dynamics of an adjustable-speed switched reluctance motor (SRM) drive with voltage pulse width modulation (PWM) regulation is carried out. Nonlinear iterative mappings based on both nonlinear and approximately linear flux linkage models are derived, hence the corresponding subharmonic and chaotic behaviors are analyzed. Although both flux linkage models can produce similar results, the nonlinear one offers the merit of accuracy but with the sacrifice of computational time. Moreover, the bifurcation diagrams show that the system generally exhibits a period-doubling route to chaos.
\end{abstract}

Index Terms-Chaos, motor drives, subharmonics, switched reluctance motors.

\section{INTRODUCTION}

I $\mathrm{N}$ RECENT years, the switched reluctance motor (SRM) drive has been recognized to have promising industrial applications. Its simplicity in both motor construction and power converter requirement offers the prospect of a low-cost high-performance brushless motor drive [1], [2]. So far, SRM research has been focused on motor design, converter topology and control strategy. To the best of the authors' knowledge, the investigation onto the nonlinear system dynamics, namely subharmonic and chaotic behaviors, in SRM drives is absent in literature. The major reason should be due to the complexity of analytical formulation and high nonlinearities of SRM drive systems.

Chaos is a common phenomenon in power converters when they operate under feedback control [3], [4]. Subharmonics and chaos in switched mode power supplies have been actively investigated for a number of years. Chaos in induction and brushless dc motor drives have also been discussed [5], [6]. Very recently, the nonlinear dynamics and chaotic behavior of industrial dc motor drives have been detailedly investigated, by both numerical simulation and analytical modeling [7], [8].

It is the purpose of this paper to investigate the nonlinear dynamics of an adjustable-speed SRM drive with voltage PWM regulation. Firstly, nonlinear iterative mappings based on both nonlinear and approximately linear flux linkage models will be derived. Then, the corresponding dynamic analysis based on a practical SRM drive system will be carried out to study the subharmonic and chaotic behaviors.

Manuscript received November 19, 1999. This work was supported in part by the CRCG of the University of Hong Kong and the Hong Kong Research Grants Council under Project HKU 7128/99E.

The authors are with the Department of Electrical and Electronic Engineering, The University of Hong Kong, Hong Kong.

Publisher Item Identifier S 0885-8969(02)01501-2.

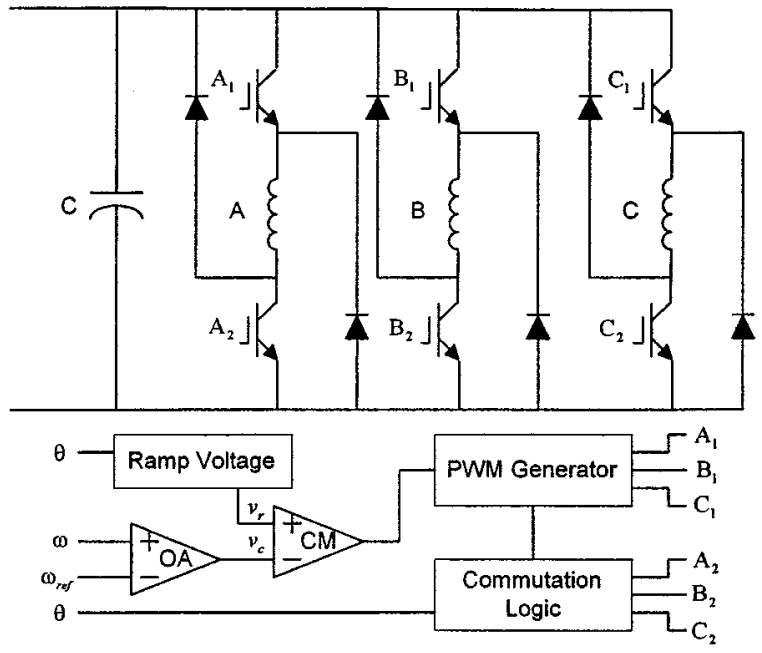

Fig. 1. Schematic diagram of SRM drive system.

\section{SYSTEM MODELING}

As shown in Fig. 1, an adjustable-speed SRM drive system is used for exemplification, where speed control is achieved through PWM control of motor phase voltages.

The SRM commutation strategy uses rotor position feedback to select the commutating parameters such as turn-on angle $\theta_{o}$ and turn-off angle $\theta_{c}$, of each phase winding of the SRM for those lower-leg power switches $\left(A_{2}, B_{2}\right.$ and $\left.C_{2}\right)$. For the sake of synchronizing the voltage PWM regulation with the phase commutation, the PWM carrier signal ramp voltage $v_{r}$, in each phase winding is a function of the instantaneous rotor displacement $\theta(t)$

$$
v_{r}(\theta(t))=v_{l}+\left(v_{u}-v_{l}\right)\left[\left(\theta(t)-\theta_{o}\right) \bmod \theta_{T}\right] / \theta_{T}
$$

where $v_{l}$ and $v_{u}$ are the lower and upper bounds of the ramp voltage, $\theta_{T}=\left(\theta_{c}-\theta_{o}\right) / n_{\theta}$ is its period, and $n_{\theta}$ is an integer.

As the op-amplifier OA has a feedback gain $g$, the speed control signal $v_{c}(t)$ can be expressed as

$$
v_{c}(t)=g\left(\omega(t)-\omega_{\text {ref }}\right)
$$

where $\omega(t)$ and $\omega_{\text {ref }}$ are the instantaneous and reference speed of the SRM. Then both $v_{r}$ and $v_{c}$ are fed into the comparator CM which outputs the signal to turn on or off those upper-leg power switches $\left(A_{1}, B_{1}\right.$ and $\left.C_{1}\right)$, depending on the phase commutation. Obviously, the interval of voltage PWM regulation of each phase winding is governed by the phase commutation. When the control voltage exceeds the ramp voltage, the upper-leg switch being the same phase of the turn-on lower-leg switch is off; otherwise it is on. The other phase switches remain off. 
Ignoring the mutual inductances among phase windings, the system equation based on the nonlinear flux linkage model is given by

$$
\left\{\begin{aligned}
& \frac{d \theta}{d t}=\omega \\
& \frac{d \omega}{d t}=\left(\frac{\partial W_{m}^{\prime}\left(\theta, i_{1}, \ldots, i_{m}\right)}{\partial \theta}-B \omega-T_{L}\right) / J \\
& \frac{d i_{k}}{d t}=\left[\frac{\partial \psi_{k}\left(\theta, i_{k}\right)}{\partial i_{k}}\right]^{-1}\left[u_{k}-R i_{k}-\omega \frac{\partial \psi_{k}\left(\theta, i_{k}\right)}{\partial \theta}\right] \\
&(k=1, \ldots, m)
\end{aligned}\right.
$$

where $u_{k}, i_{k}, \psi_{k}\left(\theta, i_{k}\right)$ are the $k$ th phase voltage, current, and flux linkage, respectively, $W_{m}^{\prime}\left(\theta, i_{1}, \ldots, i_{m}\right)$ coenergy of all $m$ phases, $R$ phase resistance, $L$ phase inductance, $B$ viscous damping, $J$ load inertia, and $T_{l}$ load torque.

The phase voltage $u_{k}$ depends on the states of its upper-leg and lower-leg power switches. If both of them are turned on, $u_{k}=U$ where $U$ is the dc link voltage; otherwise, $u_{k}=-U$ which is caused by two freewheeling diodes. If only one of them is turned on, $u_{k}=0$ which is caused by one of freewheeling diodes.

The phase current $i_{k}$ is always unipolar for the converter topology shown in Fig. 1. Moreover, the initial value of the current of each phase winding will be zero when $m>1$, since the $m$ phase windings are conducted in turn.

The phase flux linkage $\psi_{k}\left(\theta, i_{k}\right)$ is found by measurement or field analysis, which is a function of both $\theta$ and $i_{k}$. Because of high nonlinearity, it is generally expressed by an interpolation function of $\theta$ and $i_{k}$. Hence, the corresponding interpolation of

$$
\frac{\partial W_{m}^{\prime}\left(\theta, i_{1}, \ldots, i_{m}\right)}{\partial \theta}, \quad \frac{\partial \psi_{k}\left(\theta, i_{k}\right)}{\partial \theta} \text { and } \frac{\partial \psi_{k}\left(\theta, i_{k}\right)}{\partial i_{k}}
$$

can also be obtained.

Due to the switching operation, the system equation given by (3) is in fact a time-varying nonlinear state equation. By defining the state vector $\mathbf{X}$ as

$$
\mathbf{X}=\left(\theta, \omega, i_{1}, \ldots, i_{m}\right)^{\mathrm{T}} \quad \mathbf{X}\left(t_{0}\right)=\mathbf{X}_{0}
$$

the solution of (3) in the continuous-time domain can be written as

$$
\begin{aligned}
\phi_{t}\left(\mathbf{X}_{0}, t_{0}\right)=\left(\theta\left(\mathbf{X}_{0}, t_{0}\right),\right. & \omega\left(\mathbf{X}_{0}, t_{0}\right), \\
& \left.i_{1}\left(\mathbf{X}_{0}, t_{0}\right), \ldots, i_{m}\left(\mathbf{X}_{0}, t_{0}\right)\right)_{t}^{\mathrm{T}} .
\end{aligned}
$$

\section{DERIVATION OF POINCARÉ MAP}

On the investigation of subharmonic and chaotic behaviors in the drive system, the most attractive approach has been the nonlinear iterative mapping, so-called Poincaré map [4], [9].

Since SRM phase windings are conducted in turn, the sum of all $m$ phase currents $i=i_{1}+\cdots+i_{m}$ is selected as a new state variable. The new state vector becomes

$$
\mathbf{Y}=(\theta, \omega, i)_{t}^{\mathrm{T}}=\left(\theta\left(\mathbf{X}_{0}, t_{0}\right), \omega\left(\mathbf{X}_{0}, t_{0}\right), i\left(\mathbf{X}_{0}, t_{0}\right)\right)_{t}^{\mathrm{T}}
$$

which will be used to describe the dynamics of the drive system. As its commutation strategy and voltage PWM regulation are based on the rotor displacement $\theta$, the steady-state solution can be a periodic orbit with respect to $\theta$. Practically, it is impossible to find any periodic orbit in the conduction interval of each phase winding because its flux linkage also depends on $\theta$. Thus, the minimum period is at least the phase-shift angle $\theta_{s}$. A plane is defined as

$$
\Sigma:=\left\{\mathbf{Y}:\left[\left(\theta-\theta_{o}\right) \bmod \theta_{s}\right]=0\right\}
$$

The trajectory of $\mathbf{Y}$ under observation repeatedly passes through the $\Sigma$ when $\theta$ increases monotonically. The sequence of $\Sigma$ crossing defines a map

$$
P: \Re^{2} \rightarrow \Re^{2} \quad \mathbf{Z}_{n+1}=P\left(\mathbf{Z}_{n}\right)
$$

where $\mathbf{Z}=(\omega, i)^{\mathrm{T}}$.

The Poincaré map (8) is a two-dimensional (2-D) mapping, but it is based on the solution (5) of the $(m+2)$-dimensional nonautonomous equation (3). In order to avoid the calculation of the plane crossing, the rotor displacement $\theta$ rather than time $t$ is selected as the independent variable of the system. The next crossing of the plane $\theta=\theta_{o}+(n+1) \theta_{s}$ can be directly calculated by integrating from $\theta_{o}+n \theta_{s}$ to $\theta_{o}+(n+1) \theta_{s}$. To make $\theta$ an independent variable, the last $m+1$ equations of (3) is divided by its first equation and then the first equation is inverted

$$
\left\{\begin{array}{l}
\frac{d t}{d \theta}=1 / \omega \\
\frac{d \omega}{d \theta}=\left(\frac{\partial W_{m}^{\prime}\left(\theta, i_{1}, \ldots, i_{m}\right)}{\partial \theta}-B \omega-T_{L}\right) /(J \omega) \\
\frac{d i_{k}}{d \theta}=\left[\begin{array}{c}
\left.\omega \frac{\partial \psi_{k}\left(\theta, i_{k}\right)}{\partial i_{k}}\right]^{-1}\left[u_{k}-R i_{k}-\omega \frac{\partial \psi_{k}\left(\theta, i_{k}\right)}{\partial \theta}\right] \\
(k=1, \ldots, m) .
\end{array}\right.
\end{array}\right.
$$

The corresponding $v_{r}$ and $v_{c}$ can be obtained from (1) and (2) as

$$
\begin{aligned}
& v_{r}(\theta)=v_{l}+\left(v_{u}-v_{l}\right)\left[\left(\theta-\theta_{o}\right) \bmod \theta_{T}\right] / \theta_{T} \\
& v_{c}(\theta)=g\left(\omega(\theta)-\omega_{\text {ref }}\right) .
\end{aligned}
$$

Actually, only two adjacent phase windings have currents at the same time when $m>2$. For the sake of clarity and simplicity, $m$ phase windings conducted in turn can be represented by only two phase windings activated alternatively. Moreover, since the first equation of (9) does not need to be calculated, (9) can be reduced as

$$
\left\{\begin{array}{l}
\frac{d \omega}{d \theta}=\left(\frac{\partial W_{2}^{\prime}\left(\theta, i_{1}, i_{2}\right)}{\partial \theta}-B \omega-T_{L}\right) /(J \omega) \\
\frac{d i_{1}}{d \theta}=\left[\omega \frac{\partial \psi_{1}\left(\theta, i_{1}\right)}{\partial i_{1}}\right]^{-1}\left[u_{1}-R i_{1}-\omega \frac{\partial \psi_{1}\left(\theta, i_{1}\right)}{\partial \theta}\right] \\
\frac{d i_{2}}{d \theta}=\left[\omega \frac{\partial \psi_{2}\left(\theta, i_{2}\right)}{\partial i_{2}}\right]^{-1}\left[u_{2}-R i_{2}-\omega \frac{\partial \psi_{2}\left(\theta, i_{2}\right)}{\partial \theta}\right] .
\end{array}\right.
$$

Equation (12) can be rewritten as

$$
\mathbf{X}=\left(\omega, i_{1}, i_{2}\right)^{\mathrm{T}} \quad \dot{\mathbf{X}}=f(\mathbf{X}) \quad \mathbf{X}\left(\theta_{0}\right)=\mathbf{X}_{0}
$$


Therefore, the Poincaré map (8) based on (13) is given by

$$
\begin{aligned}
P\left(\mathbf{Z}_{0}\right) & =\phi_{\theta_{0}+\theta_{s}}\left(\mathbf{Z}_{0}, \theta_{0}\right) \\
& =\left(\omega\left(\mathbf{X}_{0}, \theta_{0}\right), i_{1}\left(\mathbf{X}_{0}, \theta_{0}\right)+i_{2}\left(\mathbf{X}_{0}, \theta_{0}\right)\right)_{\theta_{s}}^{\mathbf{T}} .
\end{aligned}
$$

The Poincaré map (14) is termed a nonlinear model map which corresponds to a three-dimensional (3-D) differential equation (13) including the nonlinearity of the flux linkage. Obviously, the necessary computational time in numerical simulation is lengthy.

When the drive system operates in light-load and low-peed conditions, an approximately linear flux linkage model can be adopted, which is expressed as $L(\theta) i . L(\theta)$ is given by

$$
L(\theta)= \begin{cases}L_{\min } & 0<\theta \leq \theta_{1} \\ L_{\min }+K_{l}\left(\theta-\theta_{1}\right) & \theta_{1}<\theta \leq \theta_{2} \\ L_{\max } & \theta_{2}<\theta \leq \pi / N_{r}\end{cases}
$$

where the intervals of $\left(0, \theta_{1}\right)$ and $\left(\theta_{2}, \pi / N_{r}\right)$ represent the unaligned and the aligned cases, respectively, $N_{r}$ is the number of rotor poles, and $K_{l}=\left(L_{\max }-L_{\min }\right) /\left(\theta_{2}-\theta_{1}\right)$. For each phase winding, selecting $\theta_{o}=\theta_{1}, \theta_{c}=\theta_{2}$, and $\theta_{c}-\theta_{o}$ equal to the phase-shift angle $\theta_{s}$, the electromagnetic torque in the conduction interval $\theta_{c}-\theta_{o}$ is always $K_{l} i_{k}^{2} / 2$. Assuming that $i_{k}$ gradually becomes zero within the period of $L_{\max }$, there is no electromagnetic torque produced by this phase winding in this period. Thus, $i_{k}$ in this period can be ignored since the initial value of the successive phase current must be zero. It follows that only the current of the activated phase winding will be calculated at any instance. In this case, the sum of all $m$ phase currents $i$ is discontinuous in the instance of the phase commutation. Thus, (9) is deduced as

$$
\left\{\begin{array}{l}
\frac{d \omega}{d \theta}=\left(\frac{1}{2} K_{l} i^{2}-B \omega-T_{L}\right) /(J \omega) \\
\frac{d i}{d \theta}=\frac{u-R i-K_{l} \omega i}{\left(L_{\min }+K_{l}\left(\theta-\theta_{1}\right)\right) \omega} .
\end{array}\right.
$$

Equation (16) can be rewritten as

$$
\dot{\mathbf{Z}}=f(\mathbf{Z}) \quad \mathbf{Z}\left(\theta_{0}\right)=\mathbf{Z}_{0} .
$$

Therefore, the Poincaré map (8) based on (17) is given by

$$
\begin{aligned}
P\left(\mathbf{Z}_{0}\right) & =\phi_{\theta_{0}+\theta_{s}}\left(\mathbf{Z}_{0}, \theta_{0}\right) \\
& =\left(\omega\left(\mathbf{Z}_{0}, \theta_{0}\right), i\left(\mathbf{Z}_{0}, \theta_{0}\right)\right)_{\theta_{0}+\theta_{s}}^{\mathrm{T}} .
\end{aligned}
$$

This Poincaré map is termed a linear model map which corresponds to a 2-D differential equation (16) based on the approximately linear flux linkage model.

It is important to note that the linear model map (18) facilitates to investigate the nonlinear dynamics mainly caused by the switching nonlinearity. Since (16) can be expressed as analytical formulae, it not only takes minimum computational time in numerical simulation, but is also apt to carry out preliminary analysis of nonlinear characteristics.

\section{ANALYSIS AND RESUlTS}

Based on the derived mapping, the dynamic analysis of a practical SRM drive system, in which the SRM is designed for

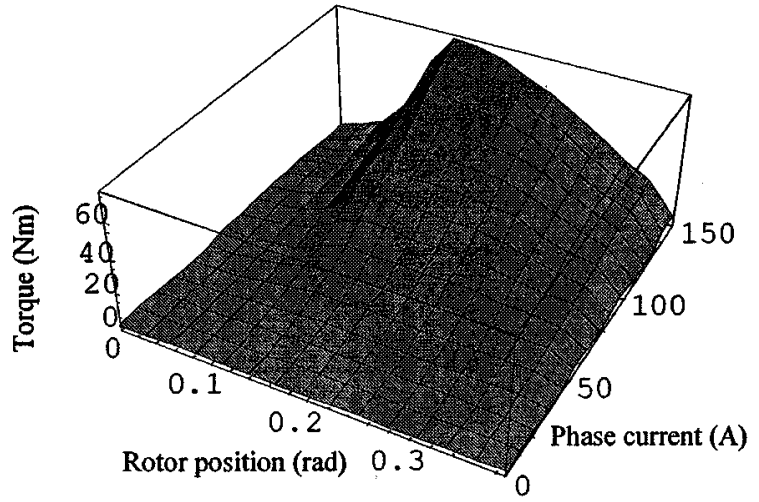

Fig. 2. Nonlinear torque characteristics.

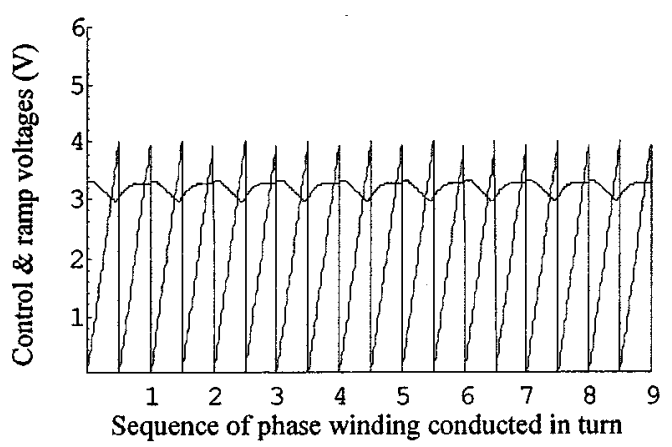

(a)

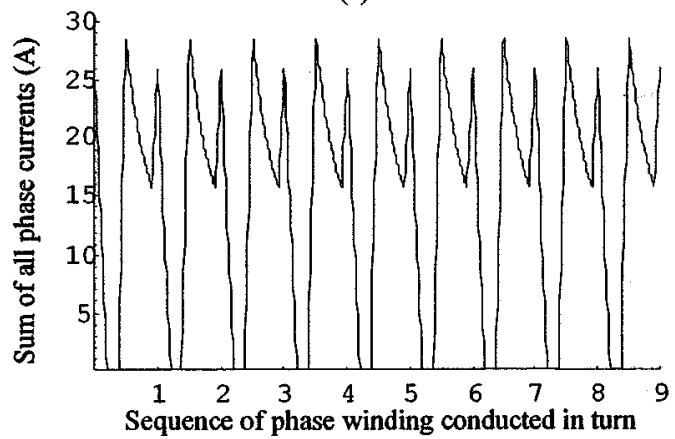

(b)

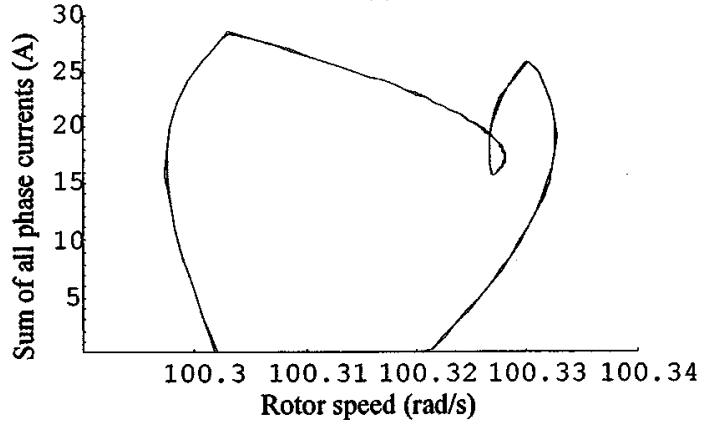

(c)

Fig. 3. Period-1 orbit. (a) Waveforms of control and ramp voltages. (b) Waveform of sum of all phase currents. (c) Trajectory of sum of all phase currents versus rotor speed.

the P-star electric vehicle [2], is carried out to study its subharmonic and chaotic behaviors.

For the sake of simplicity and clarity, two ramp voltages are adopted within the voltage PWM regulation of each phase 


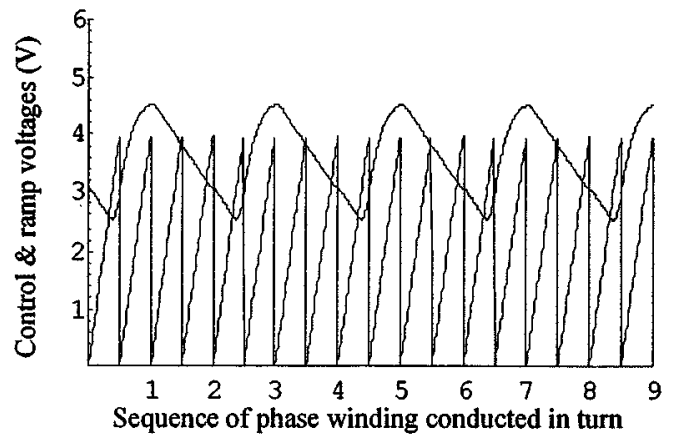

(a)

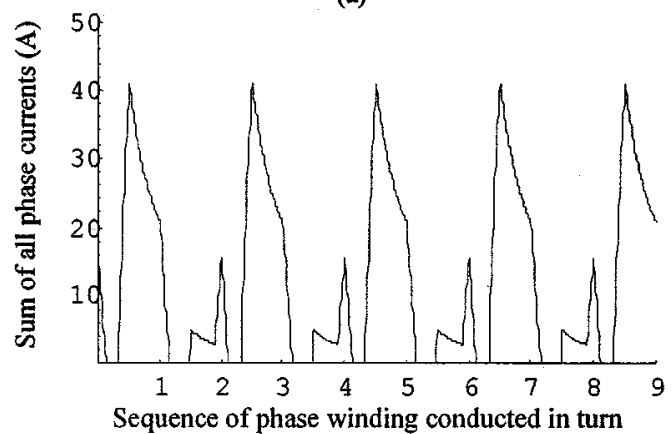

(b)

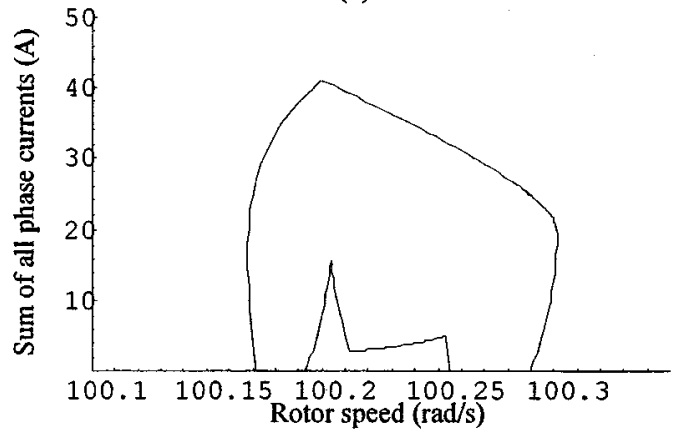

(c)

Fig. 4. Period-2 orbit. (a) Waveforms of control and ramp voltages. (b) Waveform of sum of all phase currents. (c) Trajectory of sum of all phase currents versus rotor speed.

winding. Hence, the component and parameter values of the SRM drive system are as follows:

- Controller: $n_{\theta}=2, \theta_{T}=7.5^{\circ}, v_{u}=4 \mathrm{~V}, v_{l}=0 \mathrm{~V}$, $g=10 \mathrm{~V} / \mathrm{rads}^{-1}, U=100 \mathrm{~V}, \omega_{\text {ref }}=100 \mathrm{rad} / \mathrm{s}$;

- SRM: $m=3, N_{s}=12, N_{r}=8, \theta_{1}=5.5^{\circ}, \theta_{2}=20.5^{\circ}$, $\theta_{s}=15^{\circ}, R=0.1 \Omega, L_{\min }=0.34 \mathrm{mH}, K_{l}=7.8$ $\mathrm{mH} / \mathrm{rad}, T_{l}=1 \mathrm{Nm}, B=0.0005 \mathrm{Nm} / \mathrm{rads}^{-1}, J=0.025$ $\mathrm{kgm}^{2}$ (including the inertia of the mechanical load); the nonlinear torque characteristics is shown in Fig. 2.

One way to locate a subharmonic or chaotic orbit is to integrate the system equation until the steady state is achieved. This way is called a brute-force algorithm [9]. Assuming that the Poincare map (8) reaches the steady-state orbit for the given initial values $\mathbf{Z}_{0}$ after $N_{s s}$ iteration, the $n$-point subharmonic or chaotic orbit $\left\{\mathbf{Z}_{1}^{*}, \ldots, \mathbf{Z}_{n}^{*}\right\}$ is calculated by

$$
\begin{aligned}
\mathbf{Z}_{1}^{*} & =P^{\left(N_{s s}\right)}\left(\mathbf{Z}_{0}\right) \\
\mathbf{Z}_{k+1}^{*} & =P\left(\mathbf{Z}_{k}^{*}\right) \quad(k=1, \ldots, n-1) .
\end{aligned}
$$

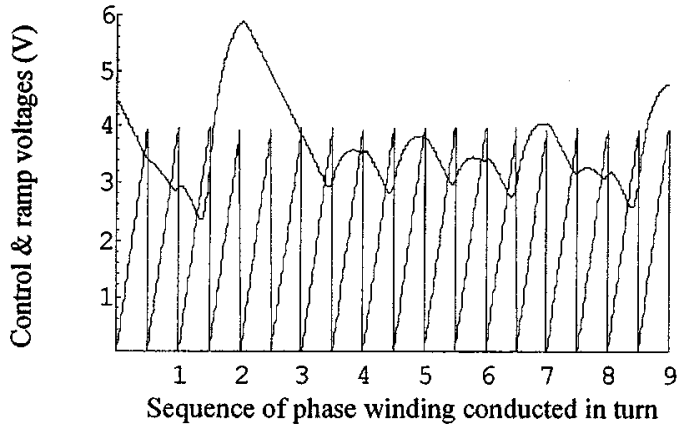

(a)

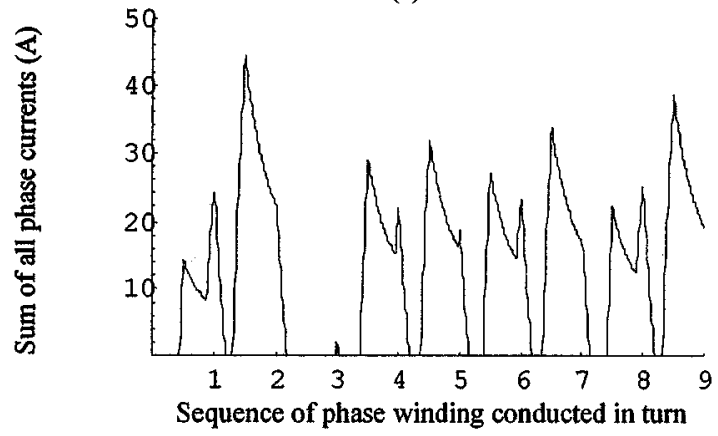

(b)

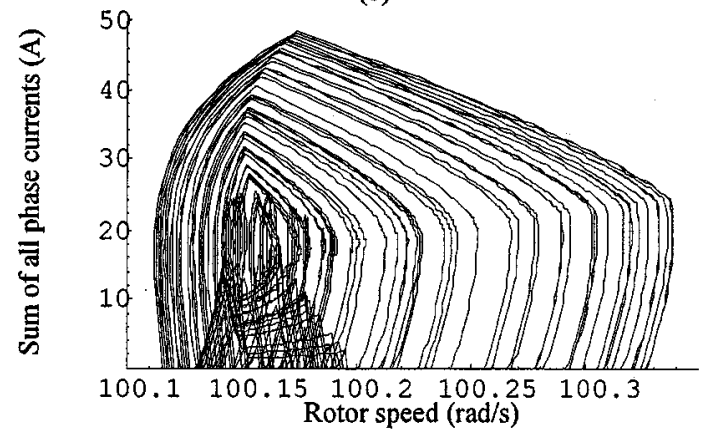

(c)

Fig. 5. Chaotic orbit. (a) Waveforms of control and ramp voltages. (b) Waveform of sum of all phase currents. (c) Trajectory of sum of all phase currents versus rotor speed.

The nonlinear characteristics of the SRM drive system will be investigated by employing this algorithm to compute the nonlinear model map (14).

\section{A. Subharmonics}

Since the current $i$ is governed by the phase commutation, the steady-state periodic solution of the SRM drive system can be a period-1 orbit $\mathbf{Z}^{*}$ or a period- $p$ orbit $\left\{\mathbf{Z}_{1}^{*}, \ldots, \mathbf{Z}_{p}^{*}\right\}$ with $p>1$ so-called subharmonics, which are described as

$$
\begin{aligned}
(1,0) \mathbf{Z}^{*} & =(1,0) P\left(\mathbf{Z}^{*}\right) \\
(1,0) \mathbf{Z}_{k+1}^{*} & =(1,0) P\left(\mathbf{Z}_{k}^{*}\right) \quad(k=1, \ldots, p-1) \\
(1,0) \mathbf{Z}_{1}^{*} & =(1,0) P\left(\mathbf{Z}_{p}^{*}\right) .
\end{aligned}
$$

For the above system parameter conditions, the steady-state solution is a period-1 orbit. The oscillation frequency of $\omega$ is about $\omega_{\text {ref }} / \theta_{s}=382 \mathrm{~Hz}$. The corresponding waveforms of $v_{c}$, $v_{r}$ and $i$ as well as the trajectory of $i$ versus $\omega$ are shown in Fig. 3 . It shows that the waveforms of different phase currents have the 


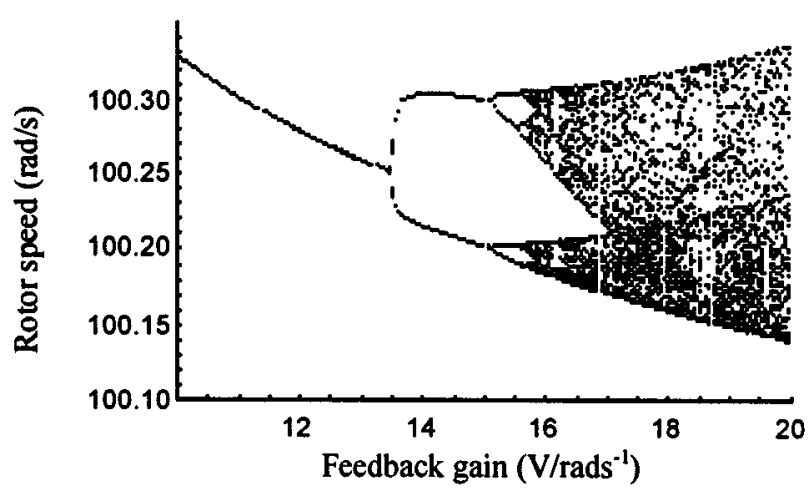

(a)

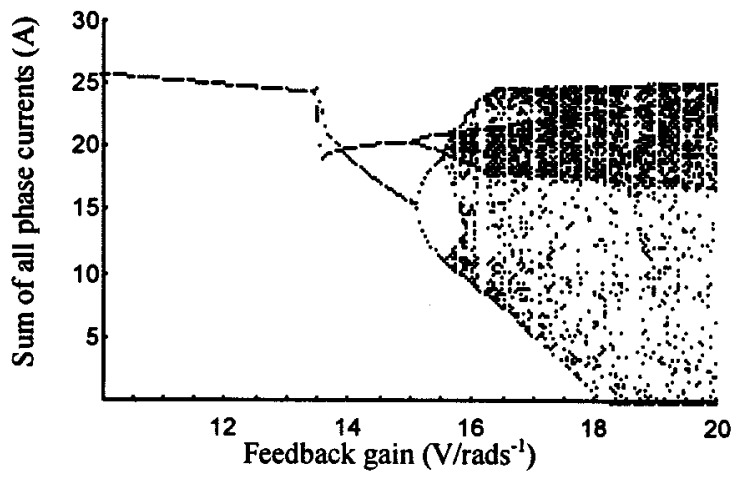

(b)

Fig. 6. Bifurcation diagrams. (a) Rotor speed versus feedback gain. (b) Sum of all phase currents versus feedback gain.

same pattern. Due to $n_{\theta}=2$, the current of each phase winding has two peaks and hence the trajectory looks like a classical period-2 orbit.

Similarly, the corresponding waveforms and trajectory of the period- 2 orbit for $g=15 \mathrm{~V} / \mathrm{rads}^{-1}$ is shown in Fig. 4 . Due to the high nonlinearity of the flux linkage, the inductance within the first ramp voltage is less than that within the second ramp. When the conduction interval within the first ramp is rather long, the current will be high enough to induce a cycle skipping phenomenon (bypassing the second ramp) as shown in Fig. 4(a). Then, the conduction interval within the first ramp of the next phase winding will be much shorter than the former. Hence, different from the period- 1 orbit, the currents of two adjacent phase windings have dissimilar patterns. Moreover, the former has the magnitude much greater than that of the latter. It should be noted that the maximum value of phase currents increases from $27 \mathrm{~A}$ of the period- 1 orbit to 40 A of the period- 2 orbit.

\section{B. Chaotic Behaviors}

Chaotic waveforms of $v_{c}, v_{r}$ and $i$ as well as the trajectory of $i$ versus $\omega$ are shown in Fig. 5. It shows that the chaotic orbit is virtually an aperiodic bounded steady-state trajectory. From the chaotic trajectory shown in Fig. 5(c), it can be found that it mainly consists of two kinds of patterns. One kind is unimodal curves while the other kind is bicorn curves. A special case of only one set of unimodal and bicorn curves is actually the period-2 trajectory shown in Fig. 4(c). Physically, the unimodal

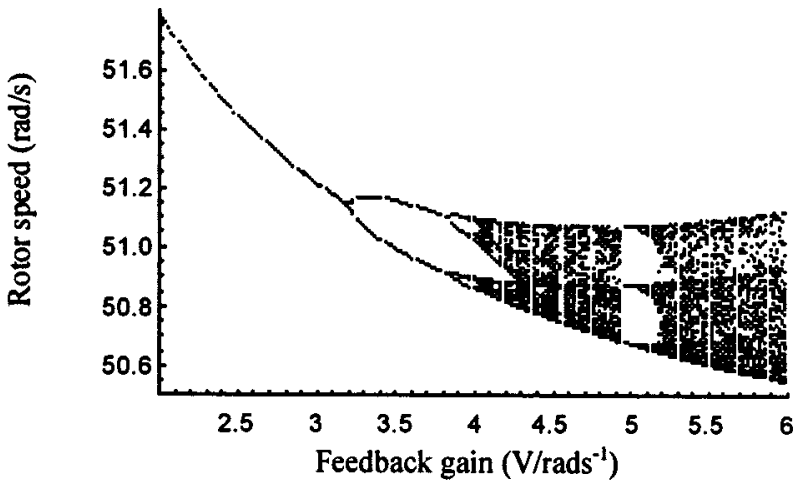

Fig. 7. Bifurcation diagram of rotor speed versus feedback gain at $50 \mathrm{rad} / \mathrm{s}$.

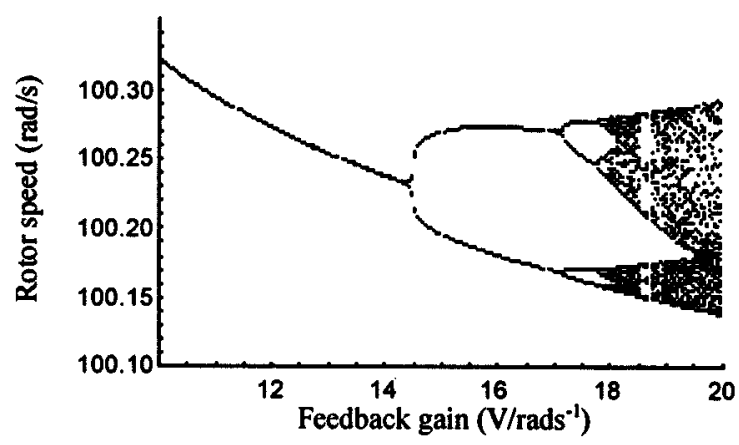

Fig. 8. Bifurcation diagram of rotor speed versus feedback gain at $100 \mathrm{rad} / \mathrm{s}$.

curve involves high current operation in such a way that the drive system tends to greatly accelerate the rotor speed within the interval of current rise, and then it will decelerate the rotor speed within the interval of cycle skipping.

Moreover, the chaotic trajectory illustrates that the trajectory with the bicorn pattern stands for stable operation while that with the unimodal pattern stands for unstable operation. Therefore, if the system trajectory can be attracted into one set of the bicorn curve, it will be a period-1 orbit as shown in Fig. 3(c); if the trajectory can be represented as one set of unimodal and bicorn curves, it will be a period-2 orbit as shown in Fig. 4(c); if the trajectory involves infinite sets of unimodal and bicorn curves, it will be a chaotic orbit as shown in Fig. 5(c). Actually, the existence of unimodal curves can be considered as a necessary condition of the occurrence of subharmonics and chaos.

\section{Bifurcation Diagrams}

For a chaotic system, it is interested in identifying how to route to chaos with respect to the variation in system parameters. As a parameter is varied, a bifurcation is an abrupt change in the steady-state behavior of the system. A plot of the steadystate orbit against a bifurcation parameter is termed a bifurcation diagram. Thus the resulting bifurcation diagrams facilitate the appraisal of the steady-state system behavior at a glance.

Based on the nonlinear flux linkage model map, the bifurcation diagrams of $\omega$ and $i$ versus $g$ for $\omega_{\text {ref }}=100 \mathrm{rad} / \mathrm{s}$ are shown in Fig. 6. When selecting $\omega_{\text {ref }}=50 \mathrm{rad} / \mathrm{s}$, the bifurcation diagram of $\omega$ versus $g$ is shown in Fig. 7. All these bifurcation diagrams illustrate that the system exhibits a period-doubling route to chaos. Comparing Figs. 6(a) and 7, it can be found 
that they are very different. Also, the corresponding value of $g$ for $\omega_{\text {ref }}=50 \mathrm{rad} / \mathrm{s}$ bifurcating into chaotic operation is much less than that for $\omega_{\text {ref }}=100 \mathrm{rad} / \mathrm{s}$. In general, the case of higher $g$ and/or lower $\omega$ is prone to exhibit subharmonic and chaotic behaviors.

Based on the linear flux linkage model map, the bifurcation diagram of $\omega$ versus $g$ is shown in Fig. 8. Comparing Figs. 6(a) and 8 , it can be found that they have similar pattern. However, the bifurcation diagram based on the nonlinear model map provides more accurate bifurcation points of subharmonics and chaos, even though the linear model map can offer faster computational speed.

\section{CONCLUSION}

In this paper, the nonlinear dynamics of SRM drives with the emphasis of subharmonics and chaos are firstly investigated. Poincaré maps (including both nonlinear and approximately linear flux linkage models) of a practical SRM drive system with voltage PWM regulation have been derived. Based on the derived maps, the characteristics of system subharmonics, chaos and bifurcation diagrams are discussed. It reveals that the system exhibits a period-doubling route to chaos. Although the investigation has been focused on a typical SRM drive, the proposed approach and derived equations can readily be applied or extended to other SRM drives.

\section{REFERENCES}

[1] P. J. Lawrenson, "A brief status review of switched reluctance drives," EPE J., vol. 2, no. 3, pp. 133-144, 1992.

[2] C. C. Chan, Y. Zhan, Q. Jiang, and K. T. Chau, "A high performance switched reluctance motor drive for P-star EV project," in Proc. Int. Elect. Vehicle Symp., 1996, pp. 78-83.

[3] J. R. Wood, "Chaos: A real phenomenon in power electronics," in Proc. IEEE Appl. Power Electron. Conf., 1989, pp. 115-123.

[4] D. C. Hamill, J. H. B. Deane, and D. J. Jefferies, "Modeling of chaotic dc-dc converters by iterated nonlinear mappings," IEEE Trans. Power Electron., vol. 7, no. 1, pp. 25-36, 1992.

[5] I. Nagy, L. Matakas, Jr., and E. Masada, "Application of the theory of chaos in PWM technique of induction motors," in Proc. Int. Power Electron. Conf., 1995, pp. 58-63.

[6] N. Hemati, "Strange attractors in brushless DC motors," IEEE Trans. Circuits Syst. I, vol. 41, pp. 40-45, Jan. 1994.

[7] K. T. Chau, J. H. Chen, C. C. Chan, J. K. H. Pong, and D. T. W. Chan, "Chaotic behavior in a simple de drive," in Proc. IEEE Conf. Power Electron. Drive Syst., 1997, pp. 473-479.

[8] K. T. Chau, J. H. Chen, and C. C. Chan, "Dynamic bifurcation in dc drives," in Proc. IEEE Power Electron. Specialists Conf., 1997, pp. 1330-1336.

[9] T. S. Parker and L. O. Chua, Practical Numerical Algorithms for Chaotic Systems. New York: Springer-Verlag, 1989.
J. H. Chen was born in Guangdong, China, in 1961. He received the B.Sc. (Eng.) and M.Sc. (Eng.) degrees in electrical engineering from Tsinghua University, Beijing, China, in 1982 and 1987, respectively. He is currently pursuing the Ph.D. degree at the University of Hong Kong.

Prior to entering the Ph.D. program, he was an Associate Professor with the Department of Electrical Engineering, Tsinghua University. His areas of interests include electrical machine and drive design, modeling, simulation, and power electronics. He has published several papers in this area.

K. T. Chau (M'89) received the first-class honors B.Sc. (Eng.), M.Phil., and $\mathrm{Ph} . \mathrm{D}$. degrees in electrical and electronic engineering from the University of Hong Kong.

Currently, he works as Associate Professor at the University of Hong Kong. His teaching and research interests focus on three main areas-power converters, machines and drives, and electric vehicles. In these areas, he has published over 100 refereed technical papers and some industrial reports. He has also served as chair and organizing committee member for many international conferences.

C. C. Chan (M'77-SM'77-F'92) received the B.Sc. degree from China University of Mining and Technology, Beijing, China, the M.Sc. degree from Tsinghua University, Beijing, China, and the Ph.D. degree from the University of Hong Kong, in 1953, 1957, and 1981, respectively.

He began his professional electrical engineering career in 1959 . He worked for 11 years in industry and 26 years in academic institutions. He is now the Head of the Department of Electrical and Electronic Engineering, the Honda Chair Professor of Engineering, and the Director of the International Research Centre for Electric Vehicles, all at The University of Hong Kong, Hong Kong, China.

Dr. Chan was awarded the Honorary D.Sc. Degree from the University of Odessa in 1992 for his contributions to the advancement of electric drives and electric vehicles. He is also a Fellow of IEE, HKIE, and the Royal Academy of Engineering. He is very active in professional society activities. He is the Senior Vice President of the Hong Kong Institution of Engineers and holds over 20 posts in international committees. He also serves as Consultant to several organizations in Hong Kong and the U.S.

Quan Jiang received the B.Eng. degree in electrical engineering from Hefei Polytechnic University, Hefei, China, and the M.Eng. and Ph.D. degrees in electrical engineering from Southeast University, Nanjing, China, in 1983, 1986, and 1991, respectively.

From 1991 to the present, he has worked as a Lecturer and Associate Professor in the Department of Electrical Engineering, Southeast University. From 1994 to the present, he has worked as a Postdoctoral Fellow and a Research Associate at the University of Hong Kong. His main research interests are in design and control of electric machines and their controllers, power electronics and finite element analysis. He has published more than 20 technical papers. 